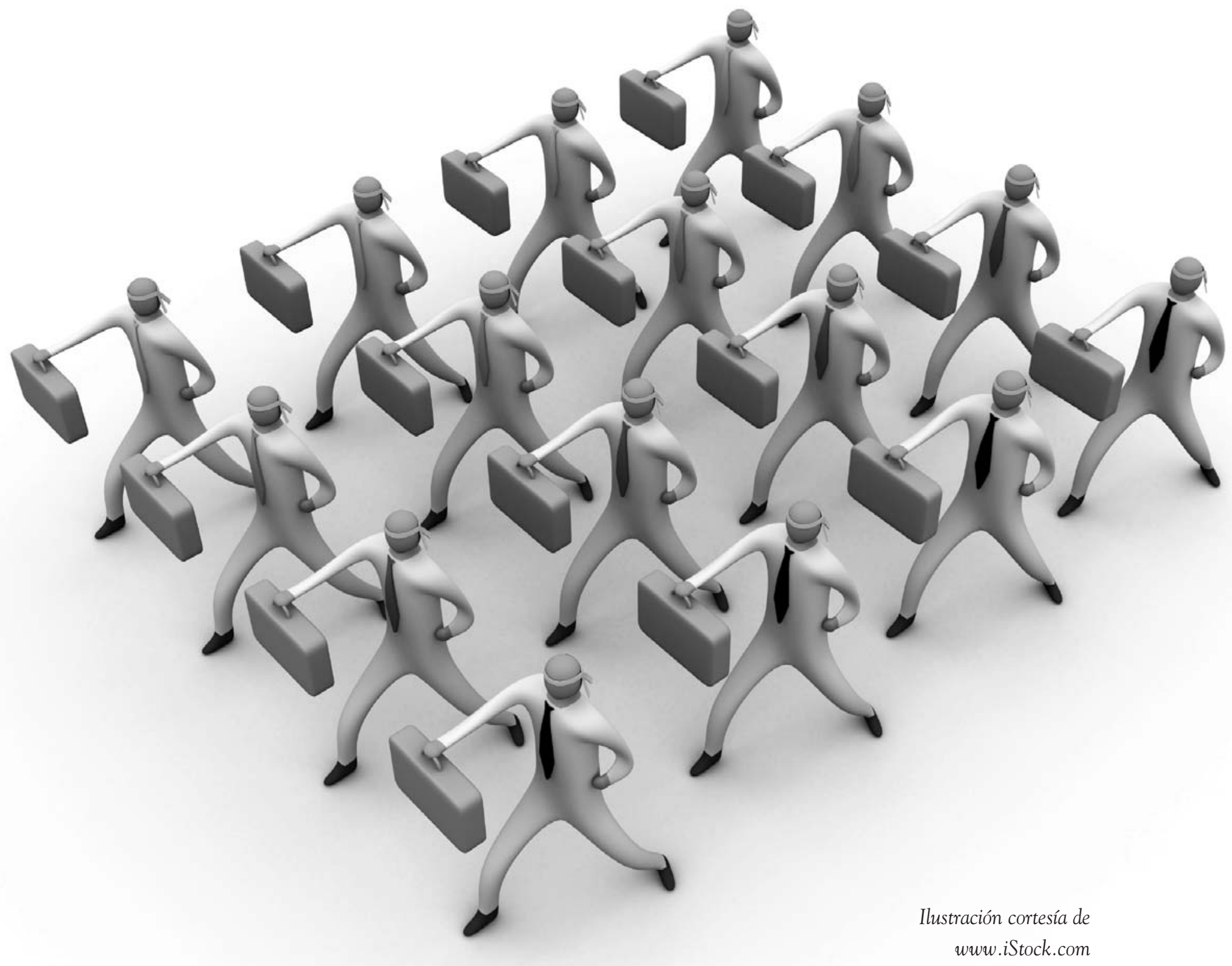

\title{
Resumen
}

El rápido crecimiento de la industria de las artes marciales justifica el profundizar en el conocimiento sobre su proceso de globalización. Hasta la fecha, existe muy poco conocimiento sobre las artes marciales como productos de consumo global y su proceso de difusión. En este artículo, los autores abordan la expansión e integración global de las artes marciales asiáticas. Se examinan en detalle factores como la deportivización y la estandarización, como fuerzas que motivan significativamente el crecimiento de la industria de las artes marciales. También se examinan algunos temas importantes, tales como el entorno político, la cultura, y las estrategias de mercado, en términos de su influencia sobre el desarrollo y el futuro crecimiento de la industria de las artes marciales. Este estudio concluye con una discusión sobre los retos que se presentan y las futuras oportunidades de desarrollo. 


\title{
La Globalización
}

\section{DE las ARTES Marciales: El Cambio de Reglas para los Nuevos Mercados}

\author{
Yong Jae Ko \& Jin Bang Yang
}

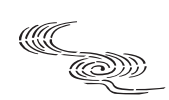

\section{Introducción}

Históricamente, las artes marciales han sido durante mucho tiempo una forma importante de actividad física y de educación en los países del este de Asia (Ko, 2002). La industria de las artes marciales ha experimentado un tremendo crecimiento y ello ha conducido a una creciente concienciación y a una mayor implicación en las artes marciales a nivel mundial (Ko, 2002, 2003). Como resultado, las artes marciales han llegado a ser una parte integral de los deportes y de las actividades físicas. Las artes marciales conducen a un estilo de vida saludable y a unos valores culturales que tienen una función tanto educativa como recreativa en muchos países occidentales. El número cada vez mayor de productos, practicantes, organizaciones, y eventos de artes marciales refleja que éstas son una parte integral de nuestra sociedad.

Hoy en día, la industria de las artes marciales está evolucionando rápidamente en un mercado maduro y altamente competitivo (Ko, 2002, 2003). En los Estados Unidos, por ejemplo, previsiones recientes han estimado que el número de participantes en artes marciales se ha incrementado en un 60\% durante la última década, de 3.6 millones de personas en 1993 a 6 millones en el 2001 (NSGA, 2002). El número de escuelas comerciales de artes marciales también se ha incrementado de 13.600 en 1999 a 14.500 en el 2003 (InfoUSA, 2003). Mientras tanto, la industria de las artes marciales afronta muchos retos para poder competir con las principales industrias tanto en el ámbito de los deportes (nuevos y tradicionales) como en el de las actividades de ocio.

A medida que las artes marciales continúan cruzando fronteras y crecen como parte de la cultura popular en la comunidad global, la literatura de las artes marciales ha reclamado una amplia investigación sobre el proceso de globalización y las estrategias que han seguido las organizaciones de artes marciales. En realidad, aunque las artes marciales se han transformado para incrementar significativamente el rango de opciones disponibles para los consumidores de las mismas en los diversos países, los patrones adoptados no siempre han sido sensibles para asegurar el éxito en la difusión del potencial educativo y recreativo de las artes marciales. Para que las artes marciales puedan sobrevivir en este entorno altamente competitivo, es esencial desarrollar una mejor comprensión sobre los factores que influyen en la globalización de las artes marciales como productos culturales y sobre los procesos de toma de decisión de los consumidores de artes marciales en diversos territorios. El esfuerzo académico a realizar para comprender estos temas es esencial no sólo para mantener el éxito actual de las artes marciales, sino también para impulsar el futuro crecimiento de su industria. Hasta hoy, sin embargo, la literatura ha descuidado el proceso de difusión de las artes marciales en la sociedad occidental. Así, aún existe un escaso conocimiento sobre las artes marciales como productos de consumo global, habiéndose realizado pocos esfuerzos en el ámbito académico para examinar estos temas. 
Consecuentemente, el propósito de este estudio es examinar las artes marciales como un producto cultural popular, proponer futuras investigaciones y examinar las implicaciones para la dirección de las organizaciones de artes marciales. Específicamente, el estudio incide en la exploración de la industria de las artes marciales mediante el examen de su proceso de globalización y sus problemas potenciales. Además, se discuten las estrategias clave para el futuro desarrollo de las artes marciales en el mercado global, así como un conjunto de obligaciones para los líderes de la comunidad de las artes marciales. Este estudio puede proporcionar a los líderes de las artes marciales importantes elementos para la dirección de las mismas, orientados a desarrollar estrategias de dirección efectivas que tengan como objeto a los practicantes de artes marciales en el mercado global. De momento, no ha existido ninguna investigación sustancial sobre este tema. El presente estudio realizará contribuciones tanto en el terreno científico como práctico.

\section{L a G loba 1 i z a c ión de las Artes Marciales}

La globalización es "el proceso directivo de integrar actividades mundiales en una única estrategia mundial mediante la dirección de una red de sucursales, afiliaciones, alianzas, y asociaciones diferenciadas pero integradas" (Tallman \& Fladmoe-Lindquist, 2002: 124). Mientras que la internacionalización (i.e., expansión/diversificación internacional) se refiere a una estrategia de mayor presencia en el ámbito internacional, la globalización (i.e., integración global) implica una estrategia de consolidación de mercados y operaciones internacionales en una única entidad estratégica mundial. La globalización es un esfuerzo estratégico para tratar al mundo como un mercado único, lo cual requiere diversas combinaciones de capacidades, estrategias y recursos (Tallman \& Fladmoe-Lindquist, 2002).

La globalización de las artes marciales asiáticas, a través de su amplia aceptación como deportes globales, ha resultado en una creciente concienciación sobre estas prácticas y en una mayor participación a nivel recreativo, amateur y profesional en todo el mundo (Ko, 2003). En particular, se ha hipotetizado que esta transición desde un arte de combate tradicional a un deporte orientado a la competición ha sido el mayor catalizador del crecimiento y popularidad de muchos estilos de artes marciales modernos (Yang, 1997).

\section{La difusión de las artes marciales asiáticas en los Estados Unidos}

Desde mediados de la década de 1880 hasta principios de la década de 1890, las artes marciales asiáticas eran practicadas en áreas limitadas de los Estados Unidos. Desde 1884 , los jornaleros chinos viajaron en tropel a las ciudades mineras de California y a los lugares de construcción de las vías férreas, y los inmigrantes japoneses emigraron a las granjas de California y Hawai. Estos inmigrantes asiáticos practicaban sus propias artes marciales tradicionales en sus comunidades (Corcoran \& Farkas, 1983; Davey, 1996).

En 1902 y 1903, los profesionales del judo japoneses Yamashita Yoshiaki y Tomita Shumeshiro visitaron los Estados Unidos, e introdujeron formalmente el judo en la sociedad americana (Corcoran \& Farkas, 1983). Sin embargo, no fue hasta después de la Segunda Guerra Mundial cuando América tuvo una exposición significativa a las artes marciales asiáticas. Cuando las tropas americanas ocuparon Japón, Corea, Okinawa, y algunas partes de China, muchos estadounidenses tuvieron la oportunidad de aprender artes marciales asiáticas, trayendo a casa dichas artes. En algunos casos, invitaron a sus profesores extranjeros a impartir sus enseñanzas en los Estados Unidos (USTU, 1990). De acuerdo con Min (1996: 4), "la versión moderna del taekwondo debe su carácter a aquellos maestros que se asociaron después de la liberación de Corea de la dominación colonial (japonesa) para establecer un arte marcial coreano que fuese capaz de trascender las fronteras nacionales y que permitiese a Corea compartir alguna de sus tradiciones más refinadas con el resto del mundo".

La globalización de las artes marciales... $\diamond$ Yong Jae Ko \& Jin Bang Yang 
En la década de 1950, se produjeron algunos eventos históricos que promocionaron el kárate japonés y el taekwondo coreano entre el público americano. En 1952, el coreanojaponés Mas Oyama recorrió los Estados Unidos para introducir el kárate al público americano; en 1953 le siguieron diez destacados instructores de artes marciales japonesas en un tour de demostraciones (Yang, 1996). La Tabla 1 presenta una breve cronología de esta temprana difusión de las artes marciales en los Estados Unidos. Las artes marciales tuvieron un auge de popularidad a partir del estreno de la película de Bruce Lee "Enter the Dragon" [Operación Dragón], en 1973, siguiendo la serie de televisión "Kung Fu" de 1972. Desde entonces, las artes marciales asiáticas han sido un tema favorito en las películas de Hollywood y en los espectáculos televisivos (Donohue, 1994; Graper, 1983). "The Karate Kid” [Karate Kid] (1984), "Best of the Best" [Campeón de Campeones] (1989), y "Crouching Tiger, Hidden Dragon" [Tigre y Dragón] (2000) son sólo unos ejemplos al respecto.

Gracias a la popularidad obtenida a partir de la temprana difusión de las artes marciales alrededor del mundo, en la década de 1970 las artes marciales asiáticas en los Estados Unidos experimentaron cambios significativos que dieron impulso a su popularización. Un cambio fue que los estudiantes americanos alcanzaron el estatus de instructor. Otro hito fue el final de la guerra de Vietnam, un hecho importante para la difusión internacional del taekwondo. Con el ejército coreano como aliado de Occidente, se habilitó una unidad de instructores de taekwondo, en primera instancia para enseñar a determinados civiles y fuerzas militares estadounidenses, pero también tuvo como resultado el difundir el arte a otros soldados americanos. Después de la guerra, los soldados que aprendieron el arte, y de un modo más significativo sus instructores coreanos, fueron a los Estados Unidos, acelerando la difusión del taekwondo que, en aquella época, era considerado únicamente un arte menor.

\section{El estatus actual de tres de las principales artes marciales asiáticas}

La globalización de las artes marciales puede ejemplificarse a través de los casos del judo, taekwondo y wushu/gongfu. Desarrollado por el Dr. Kano Jigoro en la década de 1880, el judo se difundió por todo el mundo como resultado de la emigración japonesa. El judo fue el primer arte marcial en llegar a ser deporte olímpico en los Juegos Olímpicos de Tokio 1964 (Japón). Hoy en día, la Federación Internacional de Judo (IJF) cuenta con aproximadamente 180 miembros y más de 8 millones de practicantes en todo el mundo (International Judo Federation, 2007). El gobierno japonés ha institucionalizado la educación cultural con la "visa cultural" oficial, lo que permite a los visitantes una amplia permanencia en Japón para realizar estudios culturales, incluyendo al judo y al resto de artes marciales. El gobierno japonés también ha usado las artes marciales como instrumentos para la promoción de la cultura japonesa en el extranjero.

En Corea del Sur, aunque han existido diversas formas de artes marciales, los líderes de las artes marciales coreanas escogieron el taekwondo como el arte marcial coreano definitivo en un intento por promover su desarrollo internacionalmente (IOC, 2007). Como resultado, en 1973 se creó la Federación Mundial de Taekwondo como institución legítima dirigente del deporte. La organización está constituida por las federaciones dirigentes nacionales. Actualmente, la WTF es una de las organizaciones de artes marciales más grandes del mundo, contando con miembros en 185 países (WTF, 2007). La popularidad cada vez mayor del taekwondo y su presencia en los Juegos Olímpicos del 2000 indican claramente que el taekwondo ha llegado a ser un deporte global. Sin embargo, tras el desarrollo del taekwondo moderno está el fuerte apoyo del gobierno coreano. Como pieza para reafirmar la identidad cultural, la propagación del taekwondo por todo el mundo fue concebida como una importante meta gubernamental. Hoy en día, los líderes gubernamentales enfatizan la promoción de la cultura coreana en el extranjero para desarrollar las relaciones diplomáticas bajo el movimiento de globalización (segyehwa), y el taekwondo se concibe como un medio para este desarrollo internacional (Kim, 1996). El reciente plan gubernamental para construir una mega instalación (el Taekwondo Park) se concibe como un elemento catalizador de este movimiento.

Revista de Artes Marciales Asiáticas \ Volumen 4 Número 1 (8-19) - 2009 
Mientras que los japoneses y los coreanos ya han establecido un estándar internacional para el judo y el taekwondo respectivamente, las artes marciales chinas (e.g., wushu) están sometidas a una intensa presión para acomodarse a las demandas de la modernización y de la globalización cultural (Henning, 2006). Aunque el wushu aún no ha sido aceptado como deporte olímpico, la Federación Internacional de Wushu continúa creciendo como institución dirigente mundial, con 86 asociaciones miembro en cinco continentes en el 2001. El crecimiento de las artes marciales chinas por todo el mundo también puede dar a conocer a más personas estas diversas formas de actividad física (People's Daily, 2001).

Han existido muchos eventos históricos que jugaron importantes papeles en la globalización de las artes marciales. Aunque cada disciplina marcial tiene un proceso de desarrollo único, a partir de diferentes antecedentes históricos, existen algunos factores comunes que influyen en el proceso de globalización. Entre los mismos, pero sin limitarse a, están los siguientes:

(1) la transformación de los valores del entrenamiento en artes marciales (Donohue, 1994; Ko, 2002),

(2) la modernización de los currículos de enseñanza (Yang, 1997),

(3) los esfuerzos promocionales realizados por los gobiernos de los países de origen de las artes marciales y los crecientes esfuerzos de comercialización en el negocio de las artes marciales (Ko, 2003),

(4) la expansión global de las artes marciales a través de su deportivización y formalización (Ko, 2002; Yang, 1997), y

(5) la diversificación de los productos de artes marciales tales como películas (e.g., "Karate Kid" o "El Último Samurái"), programas de puesta en forma (e.g., el Taebo de Billy Blanks), y el surgimiento de un nuevo género de eventos de artes marciales (e.g., competiciones de artes marciales mixtas).

Como se observa en los casos del judo, taekwondo y wushu, la historia moderna de las artes marciales muestra que las artes marciales como productos culturales globales han sido formalizadas e institucionalizadas a través de su deportivización (Ko, 2002). Las organizaciones multinacionales (e.g., IJF y WTF) han desarrollado estructuras organizativas, políticas y procedimientos para incrementar la efectividad y rendimiento de los asuntos cotidianos de dirección y administración. Por ejemplo, la WTF se ha desarrollado para ser una estructura global a través de la cual las organizaciones miembro (i.e., instituciones gobernantes nacionales) desarrollan programas populares y competidores de élite, que compiten en varios eventos internacionales (Juegos Olímpicos, Campeonatos Mundiales de Taekwondo, y Universiadas). Para este propósito, la organización desarrolla normas específicas y políticas con el objeto de resolver los temas del día a día y para prevenir problemas potenciales en relación a dichos temas, tales como competidores, entrenadores, árbitros, patrocinadores, programas

Ilustraciones cortesía de www.iStock.com de entrenamiento, instalaciones, equipamientos, medios de comunicación, y procedimientos diarios de dirección y administración. 


\section{La Globalización de las Artes Marciales : Problemas y Cuestiones}

Las organizaciones de artes marciales, particularmente la IJF y la WTF, dirigen su red internacional mediante afiliados, alianzas, y asociaciones diferenciadas pero integradas. La diversificación internacional y la integración global permiten a las organizaciones de artes marciales generar ingresos adicionales en muchos mercados nacionales mediante la explotación de nuevos activos y ganando nueva fuerza de mercado al incrementar su tamaño. Por otra parte, el proceso de globalización genera varias cuestiones importantes que necesitan ser resueltas. Los conflictos del valor político y cultural y las estrategias de mercado son temas clave, entre otros muchos.

\section{Autoridad y política}

A medida que cada una de las organizaciones de artes marciales crece para convertirse en una estructura deportiva internacional, a menudo son utilizadas como una función del gobierno y como un instrumento del poder y de los intereses nacionales (Calhoun, 1987). Como resultado, pueden existir conflictos políticos entre los dirigentes y las organizaciones miembro que representan las necesidades de sus mercados locales. Esto es cierto, particularmente, en las organizaciones de artes marciales en etapas de desarrollo. En este caso, es muy importante para este tipo de organizaciones de artes marciales crear una estructura organizativa que soporte una fuerte integración (centralización) entre todos los miembros afiliados. En este proceso, sin embargo, necesitan ser capaces de controlar esta integración sin perder las cualidades y valores únicos de las organizaciones miembro individuales al ignorar sus necesidades (Bartlett \& Ghoshal, 1989). A medida que las organizaciones de artes marciales maduran y crecen, se sugiere que las organizaciones deben ser capaces de descentralizar las responsabilidades operativas a subsidiarias diferenciadas. Este proceso puede reducir dramáticamente el papel de "comando y control" de la central a favor de la "coordinación y entrenamiento". En este caso, el papel de una organización de artes marciales en la dirección y el control necesita orientarse hacia la eficiencia de la "lógica de los conocimientos prácticos" (Tallman \& Fladmoe-Lindquist, 2002). Por consiguiente, estos tipos de cambio en el estilo de liderazgo resultarán en un cambio de paradigma en la dirección y en el entorno político de muchas organizaciones de artes marciales.

Tallman y Fladmoe-Lindquist (2002) señalan algunas consecuencias positivas de la configuración estratégica de las organizaciones multinacionales: a) la capacidad para generar sinergia dinámica, b) la habilidad para usar alianzas para explorar nuevos conocimientos, más que centrarse en el dominio global para proteger el antiguo conocimiento, y c) una respuesta favorable de los mercados locales hacia los productos a través del fortalecimiento. La administración central necesita desarrollar habilidades en el ámbito de la coordinación, no del control, sobre una base global. Así, la administración es responsable de determinar estándares y de construir estructuras más que de dirigir activamente las operaciones en el día a día. Como resultado, los dirigentes saben cuándo establecer estándares (e.g., información financiera) y cuándo quedarse fuera de las transacciones. También tienen que considerarse las estrategias de imparcialidad, justicia social y distribución eficaz.

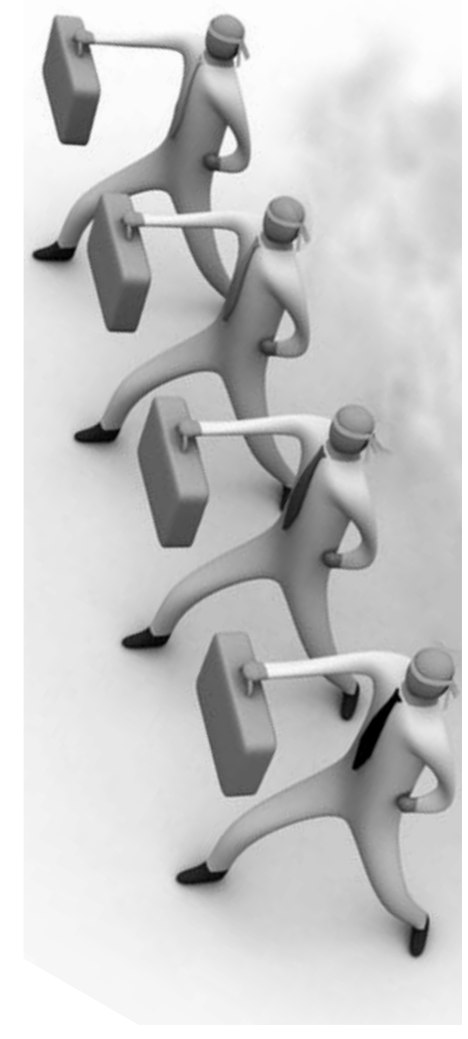

Revista de Artes Marciales Asiáticas $\diamond$ Volumen 4 Número 1 (8-19) - 2009 


\section{Cultura}

La cultura aún importa en el negocio de las artes marciales. La cultura es un conjunto de patrones de comportamiento socialmente adquiridos, trasmitidos simbólicamente a través del lenguaje y otros medios a los miembros de una sociedad particular, lo que distingue a los miembros de una categoría de la gente de otra (Wallendorf \& Reilly, 1983). La comprensión de culturas diferentes y diversas puede enriquecer el rendimiento de la organización (e.g., generación de ingresos), porque tal comprensión conduce a una penetración más viable en el mercado (Hilliard-Jones, 1996). Sin embargo, los principales problemas que afrontan los procesos de dirección internacional y transcultural proceden tanto de la diferencia de valores como de la incongruencia entre los valores centrales subyacentes en la administración central y aquellos que son centrales en el contexto cultural anfitrión (Lachman, Nedd, \& Hinning, 1994; Penaloza \& Gilly, 1999).

Considerando las artes marciales como un producto cultural popular, una de las principales metas de los dirigentes es difundir los valores culturales y el conocimiento técnico de las artes marciales entre los miembros por todo el mundo (Ko \& Pastore, 1998). En el proceso de difusión de los productos culturales, es una realidad innegable que muchos miembros de las organizaciones afiliadas pueden tener que afrontar conflictos de valores entre la cultura del mercado local y la del país de origen. Por tanto, es muy importante que los líderes de las artes marciales comprendan que pueden existir diferencias culturales fundamentales entre países y organizaciones. Además, estas diferencias pueden probablemente influir en las prácticas organizativas (Ko \& Pastore, 1998). En este caso, una fuente principal del conflicto de valores puede ser los valores confucianos de la mente oriental y el pragmatismo de los practicantes occidentales. De acuerdo con Kim (1996: 33), "el Confucianismo ve toda las relaciones sociales a la luz de una relación vertical... En una organización, esto significa que los empleados tienen que obedecer a los empleadores, y los jóvenes tienen que respetar a los veteranos". Además, el colectivismo y el individualismo (e.g., Hofstede, 1991) pueden explicar algunas fisuras culturales importantes entre los miembros internacionales. En culturas colectivistas, la individualidad se construye en términos interdependientes como una entidad conectada, relacional, de la que se espera que encaje manteniendo las relaciones interpersonales y la armonía grupal. Por otra parte, en culturas individualistas, la individualidad se construye como una entidad independiente que se espera que sobresalga diferenciándose de otras a través de los logros personales. Por ejemplo, debido a esta diferencia de valores culturales, en Norteamérica se considera generalmente apropiado enfrentarse directamente a los conflictos o problemas, mientras que en Corea y en otras culturas del Lejano Oriente, el mismo comportamiento puede percibirse como maleducado o demasiado agresivo

Ilustraciones cortesía de

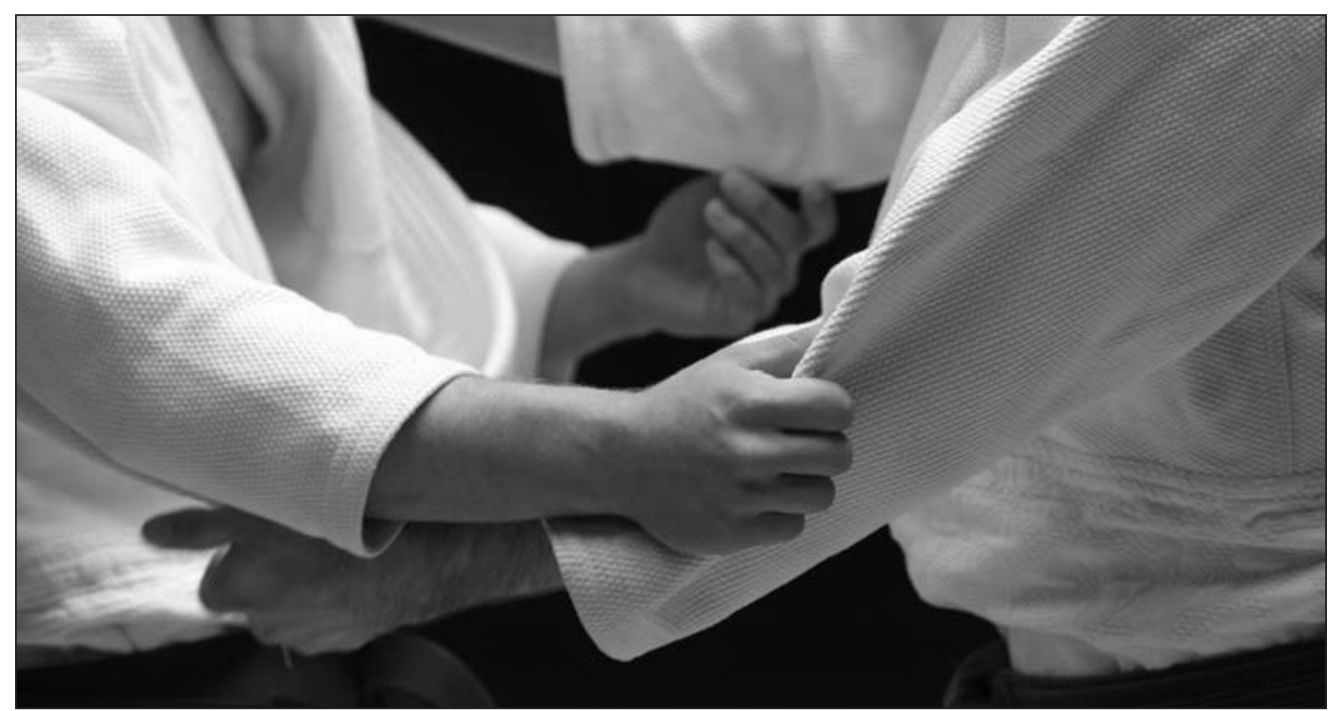

www.iStock.com

La globalización de las artes marciales... $\diamond \quad$ Yong Jae Ko \& Jin Bang Yang 
(Tushman \& O'Reilly, 1997). Otro ejemplo es que construir una relación positiva es un prerrequisito para hacer negocios en muchos países asiáticos con culturas colectivistas, mientras que, en las culturas occidentales, las relaciones comerciales tienden a ser directas y dominadas por preceptivas negociaciones legales y financieras.

Por tanto, los líderes de las organizaciones de artes marciales necesitan ser conscientes de cómo las diferencias culturales afectan al funcionamiento organizativo en los diversos mercados internacionales y cómo administrar los valores culturales y los temas relativos a la diversidad en sus organizaciones (Doney, Cannon, \& Mullen, 1998). Esto ayudará, en último término, a mejorar el rendimiento y la eficiencia en su trabajo. Aunque la estandarización y la formalización (el hardware) son muy importantes en el proceso de globalización, los líderes deberían reconocer los valores culturales únicos (el software) de los mercados locales, y ayudar a desarrollar una tercera cultura que no represente ni los valores culturales del país de origen ni la cultura del país local, a través de la difusión de las artes marciales. En realidad, aunque las artes marciales encarnan las creencias y los valores culturales de su país de origen, a través del proceso de asimilación su significado se altera gradualmente para que sean consistentes con la cultura local y logren la filiación de los participantes nativos (e.g., Duda \& Allisaon, 1990). De este modo, el entrenamiento cultural (e.g., la dirección de los recursos humanos) puede mejorar la comprensión de las diferencias culturales y ayudar a incorporar estilos particulares de dirección local y atraer los mejores talentos de todo el mundo. Los recursos humanos cualificados son los activos más importantes para las organizaciones de artes marciales y para el crecimiento de las mismas.

\section{La orientación del mercado y la confianza}

La viabilidad y el éxito financiero a largo plazo de las organizaciones de artes marciales dependen de un mejor conocimiento de los consumidores, lo que implica identificar las necesidades particulares de éstos y poder satisfacerlas proporcionando servicios de calidad. Históricamente, este tema ha despertado poco interés en esta industria, y por ello su aplicación es relativamente nueva (Ko, 2003; 2004).

La orientación del mercado es la generación de inteligencia de mercado por toda la organización y la diseminación de dicha inteligencia entre los miembros afiliados de la organización (Jaworski \& Kohli, 1993; Kohli \& Jaworski, 1990). La orientación de mercado da soporte a la filosofía de dirección que destaca la importancia central de los clientes. La carencia de orientación de mercado puede tener como consecuencias la incertidumbre sobre la demanda y errores críticos de dirección en el contexto del mercado global. Esta situación emerge cuando, por ejemplo, las organizaciones lanzan nuevos productos para los que no existe demanda latente, o cuando las organizaciones no lanzan productos que habrían tenido éxito, lo que representa una demanda latente para la cual no hay suministro (Eliashberg, Lilien \& Rao, 1997). Por tanto, las organizaciones de artes marciales necesitan continuar centrándose en quiénes son sus clientes, por qué practican artes marciales y cómo satisfacerles. La cultura organizativa centrada en el mercado y la filosofía de dirección también ayudarán a desarrollar la confianza entre los miembros de la organización.

Los practicantes de artes marciales pretenden aprender diferentes culturas y filosofías encarnadas en los valores tradicionales de las artes marciales (Ko \& Valacich, 2004). Esto indica que los consumidores perciben el entrenamiento en las artes marciales como un proceso de aprendizaje cultural. A través de este proceso, aprenden los valores tradicionales del entrenamiento de las artes marciales (e.g., respeto, perseverancia, disciplina, autocontrol, modestia, integridad, y lealtad) mientras satisfacen sus propias necesidades (e.g., beneficios físicos, mentales y sociales) (Boudreau, Folman, \& Konzak, 1995; Kennedy, 1997; Ko \& Valacich, 2004; Yang, 2000). Sin embargo, se debe realizar una investigación más sistemática para lograr una mejor comprensión del mercado global e implementar con éxito las estrategias de mercado. Esta aproximación más sistemática

Revista de Artes Marciales Asiáticas $\diamond$ Volumen 4 Número 1 (8-19) - 2009 


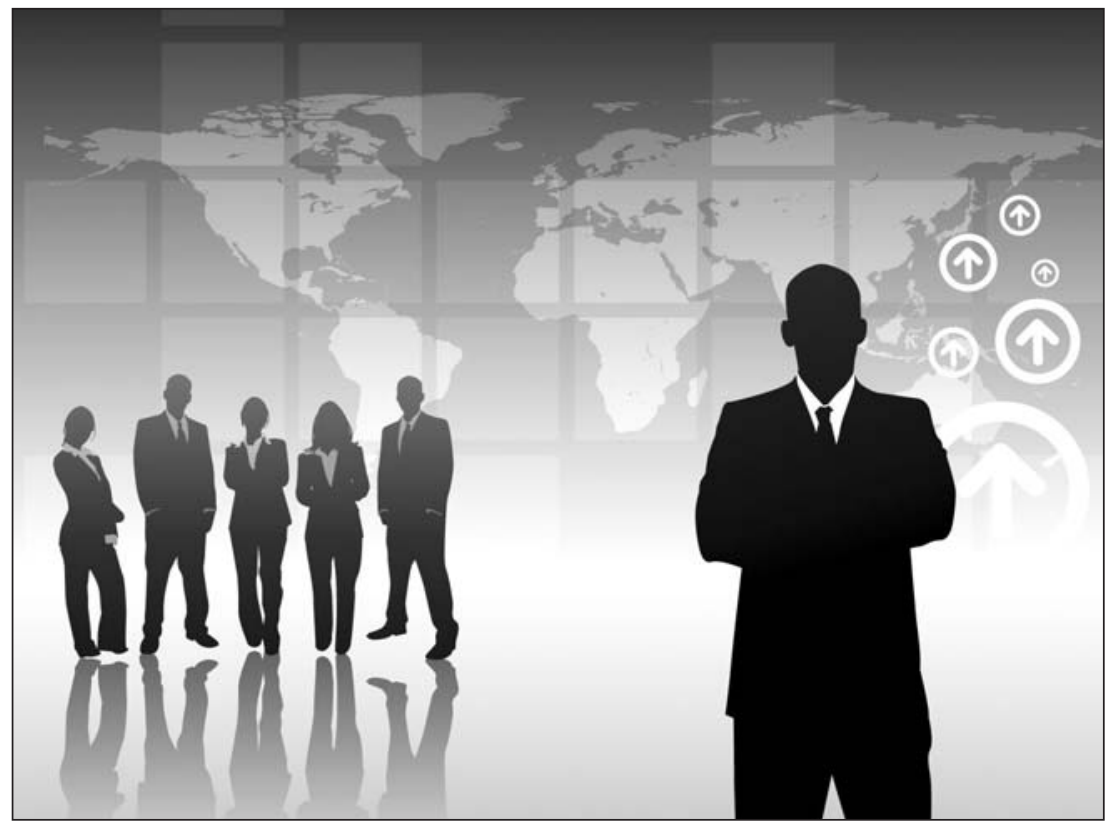

asegurará que este proceso dual de asimilación de valores culturales a través de la actividad física siga siendo la principal ventaja competitiva de las organizaciones de artes marciales (Ko, 2003).

\section{Discusión y conclusiones}

La globalización de las artes marciales continuará. Puesto que la globalización provoca cambios dramáticos en las condiciones del negocio, el predecir tales cambios resulta crucial para la preparación de respuestas por parte de las partes afectadas (Czinkota \& Ronkainen, 2005). Por tanto, el futuro éxito de las organizaciones de artes marciales depende de cómo de bien se adapten éstas a un contexto de mercado continuamente cambiante.

Ilustraciones cortesía de www.iStock.com
La última meta de las organizaciones de artes marciales es desarrollar redes para crear nuevo valor a través de la integración de varios tipos de conocimientos, recursos, y capacidades a lo largo de sus organizaciones nacionales geográficamente dispersas. Este proceso puede lograrse respetando las particularidades culturales del mercado local y la transparencia y responsabilidad de la organización. Además, la administración central en las diversas sedes centrales continuará enfrentándose a los retos de la redistribución en relación a los ingresos y al poder. Para ser un verdadero producto cultural global, la propiedad de las artes marciales debe ser compartida por igual entre los miembros. Las artes marciales ya no son propiedad de sus países de origen. Además, para conceptuarlas como un "deporte global", los líderes de las artes marciales deben centrarse no sólo en el número de naciones afiliadas (amplitud), sino también en el número de personas que participan en cada nación (profundidad), particularmente en aquellos países que están política y geográficamente aislados.

\section{Direcciones para futuras investigaciones}

Aún quedan muchas preguntas por responder. Una vez que se logre una comprensión básica de la expansión global de las artes marciales y la diversidad de su mercado, deben explorarse algunas cuestiones más específicas, como las siguientes: ¿Cómo pueden los líderes aumentar el rendimiento y la efectividad de las organizaciones de artes marciales? ¿Cuáles son las estrategias clave para promover los productos, servicios y eventos de las artes marciales? ¿Cómo afectan los factores de diversidad cultural en las estrategias para la distribución de las artes marciales en el mercado global? ¿Qué estrategias de comercialización y promoción serían más efectivas para alcanzar mercados específicos (subculturas)? ¿Hasta qué punto se satisfacen varios subgrupos pertenecientes a una nación miembro específica y cómo pueden desarrollar la lealtad, y cómo pueden las estrategias de mercado impactar en tal desarrollo? ¿Cuáles son las percepciones y actitudes que desarrolla el público en general hacia las artes marciales, sus organizaciones y eventos? y, finalmente, ¿Qué debería hacerse para promover las artes marciales como productos verdaderamente globales? El responder a tales cuestiones ayudaría en gran medida a conocer de un modo útil a los consumidores globales que participan en las artes marciales y proporcionaría a los líderes de esta industria conocimientos para el desarrollo de estrategias de mercado global.

La globalización de las artes marciales... $\quad$ Yong Jae Ko \& Jin Bang Yang 
TABLA I Breve cronología de la difusión de las artes marciales asiáticas en los Estados Unidos*

\begin{tabular}{|c|c|c|}
\hline Año & Estilo & Evento \\
\hline $1848-1863$ & Gongfu & Se importan trabajadores chinos a California para la rebatiña del oro \\
\hline $\begin{array}{l}\text { Finales década } \\
\qquad 1880\end{array}$ & Japón & Inmigrantes japoneses practican sus artes tradicionales en Hawai y California \\
\hline 1879 & Judo & El presidente de los Estados Unidos Ulises Grant visita Japón y observa la demostración de Jigoro Kano \\
\hline 1889 & Judo & Kano demuestra el judo a dignatarios americanos de visita \\
\hline 1889 & Judo & Ladd, profesor de la Universidad de Yale, estudia judo en el Kodokan \\
\hline 1902 & Judo & Yamashita Yoshiaki, primer instructor japonés de judo que enseña judo en los Estados Unidos \\
\hline 1903 & Judo & Tomita Shumeshiro enseña judo en las universidades de Princeton y Columbia \\
\hline 1907 & Judo & Ito Takugoro funda la primera escuela de judo americana en Seattle, Washington \\
\hline 1921 & & La película "Outside Women" muestra las artes marciales al público general \\
\hline 1930 & Wushu & Ark-Yuey Wong organiza un grupo de entrenamiento de gongfu en la comunidad china \\
\hline 1933 & Kárate & Higaonna Kamesuke, de Okinawa, visita Hawai \\
\hline 1934 & Kárate & Miyagi Chojun es invitado a Hawai a enseñar kárate \\
\hline 1936 & Kendo & Mori Torao emigra a Los Angeles, CA, y comienza a enseñar kendo \\
\hline 1940 & Judo & Se realiza la primera competición intercolegial de judo en San Jose, California \\
\hline 1941 & Judo & Muchos americanos practican judo en campos de relocalización japoneses \\
\hline 1946 & Kárate & $\begin{array}{l}\text { Robert Trias funda la primera escuela de kárate en la parte continental de los Estados Unidos en } \\
\text { Phoenix, Arizona }\end{array}$ \\
\hline 1949 & Judo & La Amateur Athletic Union (AAU) reconoce el judo como deporte oficial \\
\hline 1952 & Kárate & Mas Oyama realiza una gira por 32 estados haciendo demostraciones de kárate \\
\hline 1953 & Kárate & $\begin{array}{l}\text { Nishiyama Hidetaka y otros eminentes artistas marciales japoneses recorren bases militares } \\
\text { norteamericanas }\end{array}$ \\
\hline 1955 & Kárate & $\begin{array}{l}\text { Oshima Tsutomu comienza a enseñar kárate en Los Angeles, CA; la primera vez que se enseña un estilo } \\
\text { ortodoxo de kárate japonés en los Estados Unidos }\end{array}$ \\
\hline 1955 & Taekwondo & Atlee Chittim comienza a enseñar taekwondo en Texas tras volver de Corea \\
\hline 1955 & Kárate & Robert Trias organiza el primer torneo de kárate en Phoenix. Arizona \\
\hline 1956 & Taekwondo & $\begin{array}{l}\text { Jhoon Rhee se traslada a Texas desde Corea y enseña taekwondo, llegando a ser el padre del taekwondo } \\
\text { americano }\end{array}$ \\
\hline 1959 & Gongfu & Alan Lee comienza a enseñar Gongfu Shaolin en Nueva York \\
\hline 1960 & Taekwondo & Henry Cho se traslada a Nueva York para enseñar taekwondo por primera vez en la Costa Este \\
\hline 1960 & Gongfu & Bruce Lee abre su primera escuela en Seattle, WA \\
\hline 1964 & Hapkido & Choi Sea Oh introduce el Hapkido en Los Angeles, CA \\
\hline 1973 & Gongfu & $\begin{array}{l}\text { La película "Operación Dragón" de Bruce Lee se convierte en un hito en el cada vez mayor } \\
\text { conocimiento de las artes marciales asiáticas }\end{array}$ \\
\hline
\end{tabular}

* Esta tabla ha sido adaptada y levemente modificada de la disertación de Yang (1996). 


\section{BIBLIOGRAFÍA}

Bartlett, C., \& Ghoshal, S. (1989). Managing across borders: The transnational solution. Boston, MA: Harvard Business School Press.

Boudreau, F., Folman, R., \& Konzak, B. (1995). Parental observations: Psychological $\&$ physical changes in school-age karate participants. Journal of Asian Martial Arts, 4(4), 51-69.

Calhoun, D. (1987). Sport, culture, and personality. Champaign, IL: Human Kinetics.

Corcoran, J. \&, Farkas, E. (1983). Martial arts: Tradition, history, and people. New York: Galley Books.

Davey, H. (1996). Donn Draeger and International Hoplology Society. Journal of Asian Martial Arts, 5(1), 96-103.

Czinkota, M., \& Ronkainen, I. (2005). A forecast of globalization, international business and trade: report from a Delphi study. Journal of World Business, 40, 111123.

Donohue, J. (1994). Warrior dreams: The martial arts and the American imagination. Westport, CT: Bergin \& Garvey.

Doney, P., Cannon, J., \& Mullen, M. (1998). Understanding the influence of national culture on the development of trust. Academy of Management Review, 23(3), 601-620.

Duda, J., \& Allison, M. (1990). Cross-cultural analysis in exercise and sport psychology: A void in the field. Journal of Sport and Exercise Psychology, 12, 114-131.

Edwards, H. (1979). Sociology of sport. Homewood, IL: Dorsey.

Eliashberg, J., Lilien, G., \& RaO, V. (1997). Minimizing technological oversights: A marketing research perspective. En Carud, R., Nayyar, P., \& Shapira, Z., (Eds.). Technological innovation: Oversights and foresights. New York: Cambridge University Press, 214-230.

Foster, A. (1986). The nature of the martial arts and their change in the west. En S. Kleinman (Ed.). Mind and body: East meets west. Champaign, IL: Human Kinetics.

Goldstein-Gidoni, O. (2005). The production and consumption of 'Japanese Culture' in the global cultural market. Journal of Consumer Culture, 5, 155-179.

Graper, D. (1983). The kung-fu movie genre: A functionalist perspective. En S. Thomas (Ed.). Culture and communication: Methodology, behavior, artifacts, and institutions. Critical Studies in Communication, V. 3. Norwood, NJ: Ablex. 153-158.

Henning, S. (2006). China's new wave of martial studies scholars. Journal of Asian Martial Arts, 15(2), 8-21.

Hilliard-Jones, A. (1996). Consumers of color are changing the American marketplace. Marketing News, 30(24), 8.

Hofstede, G. (1991). Cultures and organizations: Software of the mind. Berkshire, UK: McGraw-Hill Co.

Jaworski, B., \& Kohl, A. (1993). Market orientation: Antecedents and consequences. Journal of Marketing, 57 (Julio), 53-70.

International Judo Federation (s.f.). The diffusion of Judo. Consulta 31 de agosto de 2007 de http://www.ijf.org/corner/qCornerView.asp?Page=1\&MenuCode= DJ\& $\mathrm{Idx}=48 \mathrm{IJF}$.

International Olympic Committee (s.f.). Taekwondo Olympic sport since 2000. Consulta 31 de agosto de 2007 de http://www.olympic.org/uk/sports/programme/ index_uk.asp? SportCode=TK

La globalización de las artes marciales... $\diamond \quad$ Yong Jae Ko \& Jin Bang Yang 
Kiм, E. (1996). A cross-cultural reference of business practices in a New Korea. Westport, CT: Quorum Books.

Ko, Y. (2002). Martial arts industry in the new millennium. Journal of Martial Arts Studies, 5, 10-23.

Ko, Y. (2003). Martial arts marketing: Putting the customer first. Journal of Asian Martial Arts, 12(2), 9-15.

Ko, Y., \& Pastore, D. (1998). Analyzing cultural values within a sport organization: The case of the United States Taekwondo Union members. The Journal of the International Council for Health, Physical Education, Sport, and Dance, 35(1), $37-41$.

Ko, Y., \& Valacich, J. (2004). Why people participate in martial arts: An analysis of motivation factors. Comunicación presentada en la Sport Marketing Association Conference. Memphis, Tennessee.

Kohli, A., \& Jaworski, B. (1990). Market orientation: The construct, research propositions, and managerial implications. Journal of Marketing, 54 (April), 1-18.

Lachman, R., Nedd, A., \& Hinnings, B. (1994). Analyzing cross-national management and organizations: A theoretical framework. Management Science, 40(1), 40-55.

Min, K. (1996, primavera). Taekwondo philosophy in the Olympic movement. USTU Taekwondo Journal, 4-5.

National Sporting Goods Association (2002). The superstudy of sports participation. Fort Mill, SC: American Sports Data.

People's Daily (2001, 29 de diciembre). Wushu seeks to become Olympic sport. Consulta 31 de agosto de 2007 de http://www.china.org.cn/english/2001/Dec/ 24469.htm.

Roberts, J. (2000). Developing new rules for new markets. Journal of Academy of Marketing Science, 28(1), 31-44.

Tillman, S., \& Fladmoe-Lindeuist, K. (2002). Internationalization, globalization, and capability-based strategy. California Management Review, 51(1), 116-135.

Tushman, M. \& O’Reilly III, C. (1997). Winning through innovation: A practical guide to leading organizational change and renewal. Boston, MA: Harvard Business School Press.

United States Taekwondo Union (1990). Taekwondo instructor manual. The United States Taekwondo Union.

Wallendorf, M., \& Reilly, M. (1983). Ethnic migration, assimilation, and consumption. Journal of Consumer Research, 10(3), 292-302.

World Taekwondo Federation (s.f.). Consulta 31 de agosto de 2007 de http://www. wtf.org/.

YANG, D. (2000). A new perspective of martial arts education for the 21st century. Journal of Physical Education, Recreation, Sport, and Dance, 36(3), 22-27

YANG, J. (1996). American conceptualization of Asian martial arts: An interpretive analysis of the narrative of Taekwondo participants. Tesis Doctoral no publicada. The University of North Carolina at Greensboro.

YANG, J. (1997). Analyzing developmental process of American martial arts school and introduction process of management system. Manuscrito inédito.

Revista de Artes Marciales Asiáticas $\diamond$ Volumen 4 Número 1 (8-19) - 2009 